\title{
Ueber die von mir an Waldeck-Rousseau vorgenommene Operation.
}

Von Prof. Dr. Hans Kehr in Halberstadt.

Der Aufforderung der Redaktion dieser Wochenschrift, eine Darstellung der Indikation und des Verlaufes der Operation, welche ich an Herrn Waldeck-Rousseau vorgenommen habe, zu veröffentlichen, komme ich um so lieber nach, als dieser durch die Tagespresse allgemein bekannt gewordene Krankheitsfall viele für den inneren Arzt,und den Chirurgen interessante Punkte aufweist, die einer eingehenden Erörterung wert sind. 
Ich bin, so oft ich über die Behandlung des Gallensteinleidens und verwandter Krankheiten geschrieben habe, immer für eine strikte Indikationsstellung eingetreten, und ich habe auch in dem Fall WaldeckRousseau tage-, ja ich kann sagen nächtelang mir genau überlegt, ob ich operieren sollte oder nicht. Ich wußte wohl, was auf dem Spiele stand. Nicht Ruhm, Ehrgeiz und Eroberungssucht haben mich über die französische Grenze geführt; ich betrachtete mich als einen Vertreter deutscher Chirurgie und deutscher Wissenschaft und hatte nur im Auge, einem Mitmenschen zu helfen, dessen Leben nur um Wochen oder Monate zu verlängern gewiß der Mühe wert gewesen wäre.

Wer meine Indikationsstellung bei der Operation des Herrn Waldeck-Rousseau verstehen will, muß über den Verlauf der Krankheit genau unterrichtet sein, er muß die diagnostischen Erwägungen kennen, die mich zur Empfehlung des chirurgischen Ein griffes bestimmten, und er muß schließlich einen Einblick bekommen in meine Operationserfolge, auf Grund deren ich die Operation anraten konnte.

Ueber den Krankheitsverlauf berichtete mir Herr Dr. Li ouville, der mich in Halberstadt aufsuchte und dann nach Paris respektive Corbeil begleitete, folgendes: Der Patient, 58 Jahre alt, früher ganz gesund, erkrankte im Spätherbst 1903 an Icterus. Dieser trat nach einer heftigen psychischen Erregung ganz plötzlich ein. Schmerzen waren nicht vorhanden. Der Icterus soll bis Frühjahr 1904 in seiner Intensität gewechselt haben. Dann stellte sich Appetitlosigkeit und Kräfteverfall ein. Im Mai 1904 wurde anderweitig eine Anastomose zwischen Gallenblase und Jejunum hergestellt. Durch einen kleinen Längsschnitt am äußeren Rand des rechten Musc. rect. abd. wurde die Bauchhöhle eröffnet, die operierenden Aerzte fühlten in der Tiefe am Pankreaskopf eine haselnußgroße Härte und legten ohne weitere Freilegung des Cysticus, Choledochus und Pankreas die Anastomose zwischen Gallenblase und Jejunum an. Die Gallenblase soll groß und verwachsen gewesen sein und ganz dicken leimartigen Inhalt enthalten haben. Die Operation verlief glatt. Patient bekam acht Tage später dreimal eine heftige (cholämische) Blutung aus der Wunde, erholte sich dann, aber der Icterus bestand fort, das Hautjucken blieb wie zuvor und brachte schlaflose Nächte. Der Appetit war immer schlecht. Das Körpergewicht war binnen Jahresfrist von 150-160 Pfund auf 110 Pfund herabgegangen. Patient befand sich zwei Monate nach der Operation, was den Icterus anlangte, auf dem Status quo ante, nur war er noch viel elender und geschwächter.

Am 8. August 1904 wurde ich konsultiert und fand einen sehr abgemagerten, stark ikterischen Patienten vor, der sehr viel schlief und schon unter den Zeichen der cholämischen Intoxikation stand. Bei der Inspektion des Abdomens zeigte sich mir eine zirka $7 \mathrm{~cm}$ lange Narbe am äußeren Rand des rechten Musc. rect. abd. Die Palpation rief nirgends Schmerzen hervor, die Leber war stark vergrößert, aber glatt und nicht sehr hart. Ascites fehlte, ein Tumor war nicht zu palpieren. Der Puls war regelmäßig und kräftig, ich zählte 84 Schläge in der Minute. Urin war frei von Eiweiß und Zucker, enthielt viel Gallenfarbstoff. Faeces waren völlig entfärbt. Die Aerzte teilten mir mit, daß Herz und Lungen völlig gesund seien; nur sei der Appetit ganz schlecht und der psychische Zustand seit einigen Tagen sehr deprimiert. Ich diagno sti zierte einen chronischen Choled ochusverschlu $B$, ob durch Tu m or (Choledochus-, Pankreas-, Duodenalkarzinom) oder durch Pankreatitis, Stein oder Adhäsionen bedingt, das war nach meiner Ansicht nicht zu entscheiden. Die beiden Chirurgen, die zuerst operiert hatten, nahmen ein Karzinom an; andere Aerzte waren der Meinung, daß bei dem kleinen Schnitt und der kurzdauernden Palpation diese sichere Annahme nur auf eine Vermutung hinausliefe, und entschieden sich für Pankreatitis chronica respektive Stein. Ich sprach mich dahin aus, daß auf Grund meiner Wirfahrungen - diese erstreckten sich zurzeit auf 947 Gallensteinlaparotomien - in $70 \%$ ein Karzinom, in $10 \%$ ein Stein in $20 \%$ eine chronische Pankreatitis vorliegen würde. Gleichzeitig stand fest, daß die drei Monate vorher angelegte Anastomose nicht funktionieren konnte, denn der Icterus war hochgradig, Urin tiefbraun, Faeces entfärbt: die erste Operation hatte also den erhofften Nutzen nicht gebracht. Woran das lag, entzieht sich natürlich meiner sicheren Beurteilung, da ich ja der ersten Operation nicht beigewohnt hatte. Aber es wurde mir berichtet, daß Adhäsionen in der Gallenblase vorgefunden wurden, und daß der Inhalt der Gallenblase sehr zâhe und fast leimartig war. Es konnte also sehr wohl der Cysticus durch Adhäsionsbildung verlegt gewesen sein, so daß also das Nicht-Funktionieren der Anastomose leicht erklärlich wird. Ich gehe, ehe ich eine Zystenterostomie ausführe, stets so vor, daß ich den Gallenblaseninhalt aspiriere. Dann wird die Gallenblase angeschnitten und der Inhalt völlig entleert. Nur wenn Galle aus dem Cysticus nachfließt, ist eine Anastomose erlaubt; scheint der Cysticus ver schlossen, so kommt entweder die Zystostomie oder besser die CholedochoDuodenostomie iu Betracht. Eventuell macht man die Ektomie und fügt eine Anastomose zwischen Choledochus und Duodenum hinzu. Jedenfalls $\mathrm{muß}$ ein hinlänglich großer Schnitt gemacht werden, damit man die Verhältnisse am Cysticus und Choledochus richtig beurteilen kann. Ich nehme an, daß in unserem Fall schon bei der ersten Operation der Ductus cysticus verlegt war, doch ist auch möglich, daß er erst nach der Operation sich verschloß. Wie dem auch sei, die Anastomose funktionierte nicht; Patient blieb gelbsüchtig wie zuvor, und die Zeichen der Leberinsuffizienz machten sich in schlimmster Art geltend. Die Angehörigen sahen das baldige Ende herannahen, sie wollten kein Mittel unversucht lassen, das Leben des Patienten zu retten Auch der Kranke selbst hatte den dringenden Wunsch nach einer zweiten Operation. Die beiden Chirurgen, die zuerst operiert hatten, hatten, so wurde mir gesagt, nichts gegen eine zweite Operation einzuwenden, wenn sie von einer auf diesem Gebiete hervorragenden Autorität als nützlich empfohlen würde. Prof. Cunéo und Dr. Liouville, die meine Veröffentlichungen über Gallensteinoperationen kannten, veranlaßten, daß ich an das Krankenbett gerufen wurde. Auch Majo Robson in London sollte geholt werden, aber da er verreist war, kam sein Assistent, Herr Dr. A rmour, nach Corbeil

Für Karzinom sprach in unserem Falle a) das Alter und das Geschlecht des Patienten (Karzinome des Pankreas kommen beim männlichen $\mathrm{Ge}-$ schlecht öfters vor als beim weiblichen); b) der fast schmerzfreie Verlauf der Krankheit (anch beim Stein möglich); c) der schwere Icterus (auch bei Stein in der Papille beobachtet); d) die Kachexie (kommt auch beim Steinverschluß vor). Gegen Karzinom sprach das plötzliche Einsetzen des Icterus, der Wechsel desselben und die relativ lange Dauer der Krankheit (fast ein Jahr). Es kommen indessen Fälle von Scirrhus des Pankreas vor, die erst nach zwei Jahren zum Exitus führen. Jedenfalls war eine absolut sichere Diagnose ganzunmöglich. Dafür stand aber das eine fest: ohne Operation war der Patient rettungslos verloren, gleichgïltig ob ein gut-oder bösartiges Lieiden vorlag. Im ersteren Fall, das heißt bei dem Befund eines Steines oder chronischer Pankreatitis, konnte die Operation, wenn sie auch keineswegs ungefährlich war, völlige Heilung bringen, während im letzteren Fall, das heißt bei dem Befund einer Neubildung, eine gänzliche Wiederherstellung unmöglich war. Aber auch hier konnte man durch eine $z$ weckmäßige Operation (Anastomose) das schreckliche Leiden erheblich lindern. Jedenfalls waren die hinzugezogenen Aerzte sämtlich der Ansicht, daß es ihre unbedingte Pflicht sei, zur Operation zu schreiten, da doch in $30 \%$ Aussicht vorhanden war, den Tod abzuwenden; aber jeder war auch darauf gefaßt (und zwar in 70\%), krankhafte Veränderungen vorzufinden, die der Kunst des Chirurgen spotten. Ja, ich hielt es nicht für ausgeschlossen, daß ich eventuell die Operation nicht bis zu Ende führen würde, da zahlreiche Verwachsungen mich daran hindern könnten. Die überaus ernste Prognose der Operation wurde aber gemildert durch den Umstand, daß die Herz- und Nierentätigkeit des Patienten noch völlig intakt war, so daß man hoffen konnte, daß der sonst sehr widerstandsfähige Patient eine zweite, im Vergleich zur ersten viel schwierigere Operation gut ïberstehen würde.

Es mußte also bei der Unsicherheit der Diagnose wenigstens eine Probeinzision gemacht werden. Konnte man sich sicher überzeugen, da\} wirklich Karzinom vorlag, so hätte man nach wenigen Minuten die Bauchhöhle wieder schließen können. Schwankte man zwischen Pankreaskarzinom und Pank reatitis chronica, so war unbedingt weiteres, Operieren geboten, das heißt, man mußte zusehen, wie man der grestanten Galle einen Ausweg verschaffen würde. Fand man einen Stein in der Papille, so kam Duodenotomie und Hepaticusdrainage in Betracht.

Ich dachte auch an eine Cholangiostomie und Cholangio-Enterostomie. Die Bedenken, die ich gegen solche Operationen hatte, hier vorzubringen, würde zu weit führen.

Ich nahm mir vor, einen genügend großen Schnitt zu machen, um mir sofort eine möglichst eingehende Orientierung zu verschaffen Patient bekam Chlorcalcium, um einer cholämischen Blutung vorzubeugen, und wurde sorgfältig zur Laparotomie vorbereitet

Da der Kranke wegen seiner großen Schwäche unmöglich transportabel war, mußte ich die Operation an Ort und Stelle ausführen. Zur Assistenz hatte ich meinen gewohnten Assistenten, Herrn Dr. R a u s c h, mitgebracht; das Instrumentarium und das Verbandzeug iöberwachte ebenfalls meine eigene Instrumentenreicherin. Die übrigen französischen Aerzte und Herr Dr. Armour (London) beteiligten sich nicht an der Operation, sondern waren Zuschauer.

Die Chloroformnarkose wurde von einem französischen Kollegen geleitet und nach der dort üblichen Art mit einem Tuche ausgeführt. Ich hätte lieber die Sauerstoff-Chloroformnarkose angewandt, aber das ließ sich nicht durchführen.

Die Operation nahm nun folgenden Verlauf : die Bauchhöhle wurde durch den Wellenschnitt eröffnet, es kam mir besonders darauf an, möglichst weit von den Verwachsungen zu bleiben, welche nach der ersten Operation entstanden sein mußten. Dabei kam mir sehr gelegen, daß der Schnitt bei der ersten Operation ziemlich weit rechts lag (siehe Fig. 1). Die Blutung aus den Bauchdecken war so, wie sie bei Cholämischen immer ist, nämlich ziemlich profus. Ueberall wurden Klemmen angelegt und größere Gefäße sofort unterbunden. Die Leber war groß, aber weich, keine Zirrhose, kein Karzinom; die Gallenblase kam mi vorderhand nicht zu Gesicht. Ich ging mit der rechten Hand in die Bauchhöhle ein und fand einen faustgroßen, ziemlich harten, mit einzelnen Knoten durchsetzten, hinter dem Duodenum gelegenen, sicher 
dem Pankreas angehörenden Tumor, von dem man mit größter W ahrscheinlichkeit annehmen konnte, dal er ein Karzinom dar-
Fig. 1.

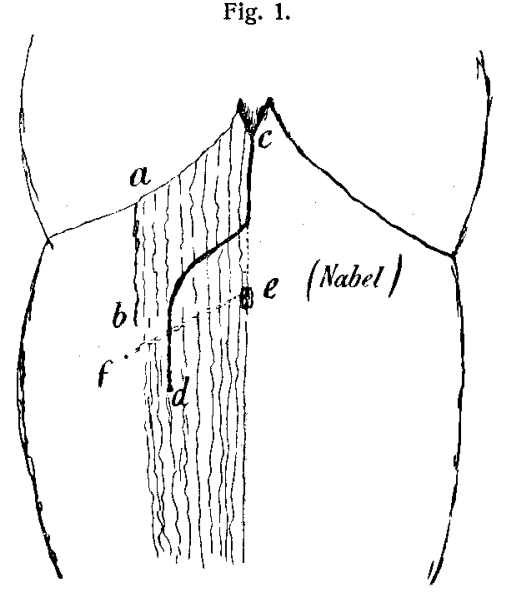
stellte. Aber da auch die chronische Pankreatitis steinharte, mit einzelnen Knoten durchsetzte Tumoren bilden kann, durfte man trotzdem die Hoffnung nicht aufgeben, daß doch nur ein chronisch entzündlicher Prozeß vorliegren würde, um so mehr, da Drüsen am Ligamentum hepato-duodenale nicht zu tasten waren und jedeSpur von Ascites fehlte. Es wäre nach meiner Ansicht falsch gewesen, wenn man schon jetzt die Bauchhöhle geschlossen hätte. Ich hielt es viel. mehr für meine Pflicht den Versuch zu machen, die gestaute Galle abzuleiten, da nur so eine Heilung eintreten konnte.

In der Tagespresse ist berichtet worden, daß eine ingeheure Blutung den Patienten dahingerafft hätte; davon weiß ich nichts. Die Blutıng bewegte sich in Anbetracht der Cholämie in ganz normalen Grenzen, und als ich die Bauchhöhle schloß, blutete kein Gefäßj mehr Dann hat man die Mär verbreitet, daß ich die krebsige Bauchspeicheldrüse entfernt hätte. Davon konnte natïrlich keine Rede sein. Es kam lediglich darauf an, die retinierte Galle abzuleiten. Das war auf verschiedene Weise möglich: entweder man machte eine Anastomose zwischen Choledochus ind Duodenum oder eine solche zwischen Gallenblase und Choledochus. Da nach dem ganzen Verlauf der Cysticus verschlossen sein muBte, wäre auch eine Anastomose zwischen Gallenblase und Hepaticus in Betracht gekommen (siehe Fig. 2). Es schien mir

Fig. 2.

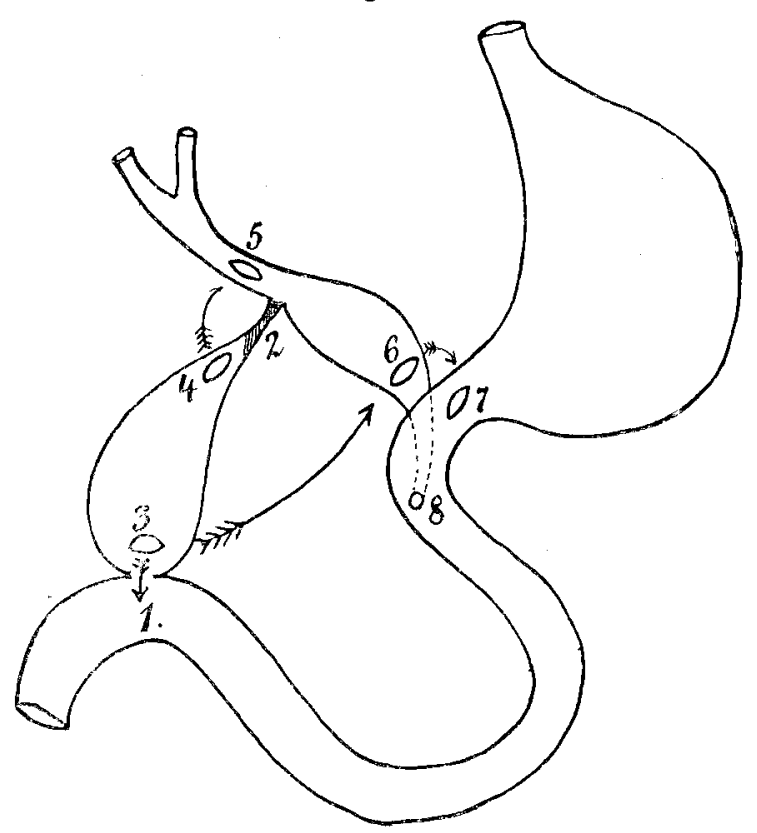

das Richtige, mich zuerst nach der vor drei Monaten angelegten Anastomose umzusehen. Von der Gallenblase aus konnte man dann das Ligamentum hepato-duodenale und den Choledochus freilegen. Ein direktes Losgehen auf den Choledochus ohne vorherige Orientierung an der Hand der freigelegten Gallenblase war wegen der dichten Verwachsungen von vornherein ausgeschlossen.

Ich suchte also die Gallenblase frei zu präparieren. Aber das war leichter gedacht als getan, und es war eine sehr mühsame, zeitraubende und schweißtreibende Arbeit, durch die festen und ausgedehnten Verwachsungen hindurch auf die Anastomose vorzudringen. Zuerst täuschte der rechte kugelige, sehr weiche Leberlappen die Gallenblase vor; ich punktierte und inzidierte ihn an einer frei gemachten Stelle, sah dann aber ein, daß ich nicht die Gallenblase vor mir hatte. Die zirka $1 \mathrm{~cm}$ lange Inzision des Lebergewebes hat natürlich dem Patienten nichts geschadet, immerhin wurde die Operation dadurch um fünf Minuten verlängert. Endlich, nach langem Suchen, fand ich die Gallenblase; es war deshalb so schwer gewesen, weil sie fast völlig leer und schlaff war. Ich sah jetzt deutlich die Anastomose und eröffnete dicht an derselben die Gallenblase. Sie enthielt trübes Sekret in geringer Menge und den Rest eines Stückchens unverdanter Melone. Die Darmfistel war offen, ziemlich eng, aber die Anastomose hatte keinen Zweck, denn der Cysticus war verschlossen. Die Lösung der Verwachsungen hatte mich ziemlich lange Zeit (1 Stunde) in Anspruch genommen. Patient fing an, etwas zu kollabieren. Es kam alles darauf an, die Operation nach Möglichkeit zu beschleunigen. Ich legte also in die eröffnete Gallenblase ein Rohr ein, verschlol dieses wasserdicht und eröffnete den fast zwei Finger starken Choledochus im supraduodenalen Teil. Im Bogen spritzte völlig wasserklare Galle hervor. Durch die plötzliche Entlastung der Leber mag der Kollaps noch stärker geworden sein, der Narcotiseur trieb zur Eile. Es war unmöglich, die geplante Choledocho-Duodenostomie oder eine Anastomose zwischen Gallenblase und Choledochus zu beenden; ich mußte mich mit der Hepaticusdrainage begnügen, tamponierte auf die gewöhnliche Weise und verschloß die Bauchhöhle. Die Operation hatte 11/2 Stunden gedauert. Sie war wegen der ausgedehnten Verwachsungen eine der schwierigsten, die ich je ausgeführt habe. Patient wurde ins Bett gebracht, der Puls erholte sich etwas, nach einer Stunde schwand er wieder.

Trotz reichlicher Exzitantien, Infusionen von Kochsalzlösung etc. trat der Exitus zirka 1\% Stunden post operationem ein.

Der Fall ist in vieler Beziehung interessant: 1. war der Krankheitsverlauf kein sehr typischer. Ein sogenannter Schulfall lag nicht vor;

Die Diagnose blieb bis zum letzten Augenblick unsicher.

Da die Möglichkeit eines gutartigen Tumors niemand ableugnen konnte, war jedenfalls eine Operation gerechtfertigt. Aber auch dann, wenn man mit Bestimmtheit ein Karzinom diagnostiziert hätte, ließ sich gegen einen Eingriff nichts einwenden. Früher war ich in dieser Hinsicht anderer Meinung. Ich hielt die operative Behandlnng des Karzinoms z. B. der Gallengänge für völlig nutzlos und nur dazu geeignet, die Gallensteinchirurgie in Mißkredit zu bringen, denn die zahlreichen Todesfälle würden manchen von der notwendigen Gallensteinoperation zurückschrecken. Ich habe meine Ansichten in dieser Hinsicht wesentlich geändert. Denn erstens gelingt die Radikaloperation eines Karzinoms dann und wann einmal doch (Körte, Majo Robson und ich verfügen über Dauerheilungen nach Karzinomoperationen an Gallenblase und Gallengängen), und zweitens läßt sich die chronische Pankreatitis von Pankreaskrebs kalum unterscheiden. Operiert man grundsätzlich beim Tumorverschluf des Choledochus nicht, so wird man manchen Menschen zugrunde gehen lassen, dem leicht zu helfen war, weil er eben an einfacher Pankreatitis litt. Deshalb operiere ich jetzt immer, in der stillen Hoffnung, statt auf ein Pankreaskarzinom auf die chronische Pankreatitis zu stoßen. Und ist das, was ich am Pankreaskopf taste, wirklich ein Karzinom, so kann ich immer noch durch eine gntangelegte Anastomose das schreckliche Leiden wesentlich lindern. Stirbt Patient bei der Operation, so ist der Tod für ihn eine Erlösung von einem kläglichen Dasein.

In der Indikationsstellung zur Operation in den in Frage kommenden Fällen geben schlieBlich das technische Geschick des einzelnen sowie seine chirurgische Begabung und seine bisherigen Erfolge den Ausschlag.

Ich hatte schon so viele Gallensteinoperationen ausgeführt, daß ich auch vor dem Fall Waldeck-Rousseau, dessen Schwierigkeiten ich keineswegs unterschätzte, nicht zurückzuschrecken brauchte, und ich konnte meiner durch große Uebung erworbenen Technik wohl zutrauen, der Situation Herr zu bleiben. Zudem gaben mir die bisher erzielten Erfolge nicht nur das Recht, sondern machten es mir auch zur Pflicht, wenigstens den Versuch einer Operation zn machen. Ich ließ mich dabei nicht von dem Wunsche des Patienten und seiner Angehörigen leiten, die natürlich alles daransetzten, dem Kranken das Leben zu erhalten, sondern lediglich meine Erfahrungen und meine Erfolge haben mein Handeln bestimmt.

Unter den französischen Aerzten war die Statistik meiner Operationserfolge bei der Cholelithiasis aus dem Handbuch der praktischen Chirurgie von v. Bergmann, v. Mikulicz, v. Bruns bekannt. Dort hatte ich meiner Statistik 720 Fälle zugrunde gelegt. Seitdem - im Verlauf von zirka zwei Jahren — habe ich weitere 227 Gallensteinoperationen ausgeführt und verfügte, als ich meine Konsultationsreise nach Paris antrat, genau über 947 Fälle. Dabei hatte ich folgende Resultate:

1. 267 konservative Operationen (Zystostomie, Zystoendyse, Zystikotomie) mit 5 Todesfällen . . = zirka $2 \%$

2. 204 Ektomien mit 7 Todesfällen . . . . . . . = $3,4 \%$

3. 191 Choledochotomien respektive Hepaticusdrainagen mit 10 Todesfällen ........ . = $5,3 \%$

4. 167 gleichzeitige Operationen wegen gutartiger Komplikationen am Magen, Darm, Leber, Pankreas mit 29 Todesfällen

5. 118 gleichzeitige Operationen wegen bösartiger Komplikationen (Karzinom der Leber, der Gallenblase, des Choledochus, des Pankreas, diffuser eitriger Cholangitis) mit 99 Todesfällen................ . . . . . . $84 \%$

Summa: $\overline{947 \text { Laparotomien mit } 150 \text { Todesfällen }=16 \% \text { Unter Abzug }}$ von 4 und 5 :

662 reine Gallensteinoperationen mit 22 Todesfällen $=3,3 \%$ 
Hatte unser Patient also einen Stein, so betrug die Gefahr der Operation $5,3 \%$, hatte er neben dem Stein chronische Pankreatitis, so mußte man mit zirka $20 \%$ Mortalität rechnen, und hatte er Karzinom, so war eine Sterblichkeit von $84 \%$ anzusetzen. Da die Diagnose unsicher war, rechnete ich, da bereits die Zeichen der Cholämie sich geltend machten, mit einer Durchschnittsmortalität von $66 \%$.

Operierte man nicht, so betrug die Sterblichkeit $100 \%$; operierte man, so war in $34 \%$ A ussicht vorhanden, das Leben der Patienten zu erhalten, und wenn ich in solchen Fällen von 100 Kranken nur drei oder vier rette, so ist das bei einem Leiden, welches ohne Operation zum sicheren Tode führt, gerade genug. Ich habe schon mehr solche Fälle operiert, die genau so elend waren wie Herr Waldeck-Rousseau, und ich habe größere Eingriffe an ihnen vorgenommen, und sie sind doch gesund geworden. Hat man auf Grund seiner Erfahrungen einerseits die feste Ueberzeugung gewonnen, daß Patient sicher verloren ist, und hat man anderseits die Aussicht, durch die Operation einen auch noch so geringen Prozentsatz durchzubringen, so wäre es Pflichtvergessenheit, die Operation zu unterlassen. Waldeck-Rousseau hatte nichts zu verlieren, er konnte nur gewinnen. Wäre die Operation gänzlich aussichtslos gewesen, so hätte ich sie ganz gewiß unterlassen. Ich bin als Chirurg und nicht als pathologischer Anatom nach Frankreich gefahren. Das mögen sich diejenigen merken, die behauptet haben: Die Operation hätte nur den Zweck gehabt, die von französischen Aerzten schon lange vorher gestellte Diagnose Krebs des Pankreas zu erhärten. Meine Operation hatte in erster und letzter Linie einen kurativen $Z$ weck!

Unsere chirurgischen Eingriffe, gerade diejenigen, die einer vitalen Indikation entspringen, gehen nun einmal "auf Leben und Tod“. Und wenn es auch richtig ist, daß der unvermeidliche Blutverlust, die Einwirkung des Chloroform auf das Herz, die lange Dauer der Operation das Ende beschleunigt haben, so * ist die eigentliche Ursache des ungünstigen Ausganges doch in der Grundkrankheit) zu suchen, die zu Leberinsuffizienz und cholämischer Intoxikation führte.

Unser Fall ist weiterhin dadurch bemerkenswert, da 3

2. die vor drei Monaten angelegte Anastomose nicht funktionirte. Wie man derartigen unangenehmen Vorkommnissen vorbeugen kann, geht aus den obigen Bemerkungen hervor.

3. Der Fall hat mir wiederum gezeigt, wieviel besser ein Patient, der sich einer Gallensteinoperation unterziehen will, in einem Hospital aufgehoben ist. Die Angehörigen und die behandelnden Aerzte haben in rührender Sorge nichts fehlen lassen, um den Erfolg der Operation zu garantieren, und ich selbst glaubte für alles gesorgt zu haben: eigenes Instrumentarium, eigene Assistenz. Und doch fehlte mir mancherlei: Oberlicht, verstellbarer Operationstisch, Sauerstoff-Chloroformapparat. - Deshalb soll man nur in der dringendsten Not - diese lag allerdings hier vor! - sich zu einer Operation im Privathause bestimmen lassen.

Herr Waldeck-Rousseau ist tot; ich als deutscher Chirurg hätte gern in dieser Wochenschrift von einer glücklichen Operation an diesem großen französischen Staatsmann berichtet; doch so glanzvoll sich auch die Bauchchirurgie, speziell die der Leber und Gallenwege, entwickelt hat, bei der weit voroeschrittenen Krankheit und der großen Schwäche des Patienten kann es nicht verwundern, daß die auf Grund vitaler Indikation ausgeführte Operation nicht den erhofften Erfolg gehabt hat. 